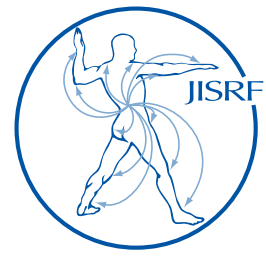

\title{
The iDuo Bi-compartmental Knee Replacement: Our Early Experience
}

Jemmett $P^{1}$, Roy $S^{1}$

\section{Abstract}

We present the first UK single surgeon case series for the iDuo knee. This is a CT based custom fit monolithic bi-compartmental design that resurfaces both trochlea and condyle. Perceived benefits include maintenance of normal kinematics and preservation of bone stock on the unaffected side. The femoral component is tailored to the patient with no compromise of either the trochlea or femoral geometry.

Method: Patients were selected based on functional ability and physiological age who had an intact symptom free lateral compartment. Knee Society scoring (KSS) was performed pre-operatively and at regular intervals. Patients were asked whether they would undergo the same operation at the one-year mark.

Results: Seven patients have undergone this procedure from 2013 until present. Average age is 60 (Range 55- 82). Average pre-op KSS was 108. All patients consistently scored higher at each interval follow up with excellent results at one year (Av KSS 194). This benefit was seen past two years in all but one in those reaching this point.

Conclusion: Our early results suggest that the iDuo knee is a good option for those with isolated bicompartmental disease and outcome scores are comparable with those reported for the BKA. This bicompartmental design may bridge the gap between the uni-compartmental and total knee replacement. The choice between monolithic or modular designs remains in debate. We will continue to use this prosthesis for a carefully selected group of patients.

Keywords: knee; arthroplasty; bi-compartmental; monolithic Level of Evidence: AAOS Therapeutic Level IV

\section{Introduction}

Even today, it is not uncommon for patients with isolated compartmental wear to be treated with total knee replacement. Whilst an acceptable result is obtained in many cases, it seems unnecessary to sacrifice healthy bone stock and the anterior cruciate ligament when all three compartments are not affected. There has been a resurgence in the use of uni-compartmental replacement granting a select
1 Dr Peter Jemmett, Dr Stuart Roy Royal Glamorgan Hospital, Cwm Taf University Health Board, United Kingdom (Direct reprint requests to Peter Jemmett)

(C) 2016 Jemmett, Roy. All rights reserved.

Reconstructive Review is a peer-reviewed, open-access orthopaedic journal devoted to publishing papers in the area of reconstructive arthroplasty. Authors retain copyright and grant the journal right of first publication with the work. Reconstructive Review follows the Creative Commons Attribution-NonCommercial CC BY-NC. This license allows anyone to download works, build upon the material, and share them with others for non-commercial purposes as long as they credit the senior author, Reconstructive Review, and the Joint Implant Surgery \& Research Foundation (JISRF). 
group of patients a better chance of returning to normal functional activity and low impact sports $[\underline{1}, 2]$. Unfortunately, medial compartmental structural cartilage damage will often progress to the patella-femoral joint [ $\underline{3}$ ] and given the strict criteria in most centers, UKA for these younger patients with some additional patella-femoral involvement may not be appropriate [4].

The use of bi-compartmental knee arthroplasty (BKA) to address this problem is in its infancy. Data for these is sparse and reports are mixed. Parratte et al [5] retrospectively analyzed a patient group who had undergone patella-femoral and unit-condylar arthroplasty in combination. Functional scores at a mean of 12 years were encouraging but implant survival was $54 \%$ at 17 years. Recent short term results using combinations of commonly used implants are promising $[\underline{6}, \underline{7}, \underline{8}]$ and yet studies exist to suggest no benefit of bi-compartmental arthroplasty over total knee replacement when considering functional outcome [9].

The Journey-Deuce bi-compartmental prosthesis (Smith \& Nephew, Memphis, Tenn) was introduced in 2005. This employed a single femoral component (monolithic), which resurfaced the medial femoral condyle and trochlea groove. Unfortunately tibial plate loosening and inconsistent pain relief led to its withdrawal $[10,11]$.

More recently, ConforMIS (Bedford, MA) has developed the iDuo monolithic partial knee replacement for bicompartmental disease affecting the medial or lateral tibio-femoral and patella-femoral joints. This system seen radiologically and graphically in Figure 1, employs single use jig instrumentation customized for each patient following pre-operative CT scanning. Again the femoral component resurfaces both the condyle and trochlea groove. The perceived benefits, as with the Journey-Deuce knee include maintenance of normal kinematics, preservation of bone stock on the unaffected side and preservation of both cruciates. Unlike the Deuce knee however, the femoral component is tailored to each patient with no com- promise of either the trochlea or femoral geometry. In addition, operating room efficiency may be improved with pre-sterilized disposable instruments and a single reusable instrument tray.

This study is the first short term outcome study for the iDuo knee and aims to investigate whether we now have a viable monolithic option to bridge the gap between unicompartmental and total knee replacement (TKR).

\section{Method}

Patients were listed for this procedure provided that they had established medial and patella-femoral compartment osteoarthritis with an intact lateral compartment. Examination and plain films in tandem were employed to make this decision. Whilst age was not used as an absolute exclusion factor we felt it important that patients should lead an active lifestyle without significant co-morbidity. Patients then underwent mapping CT scanning within 3 months of the procedure. Patient specific jigs and prostheses were manufactured accordingly. A final decision to perform bi-compartmental resurfacing rather than total replacement was made at the time of surgery with full evaluation of all compartments via a midvastus approach. All patients that were scheduled for bi-compartmental surgery were implanted with the iDuo; there were no patients who were converted at time of surgery.

All patients underwent Knee Society Scoring (KSS) on the day of surgery and at 6 weeks, 3 months, 6 months, 1 year and annually from then on where possible. This system scores the knee itself and the overall function of the patient. A Knee or function score of 80-100 can be classified as excellent, 70-79 as good, 60-69 as fair and below 60 as poor. At one year, patients were asked whether they would be prepared to undergo the same procedure again.
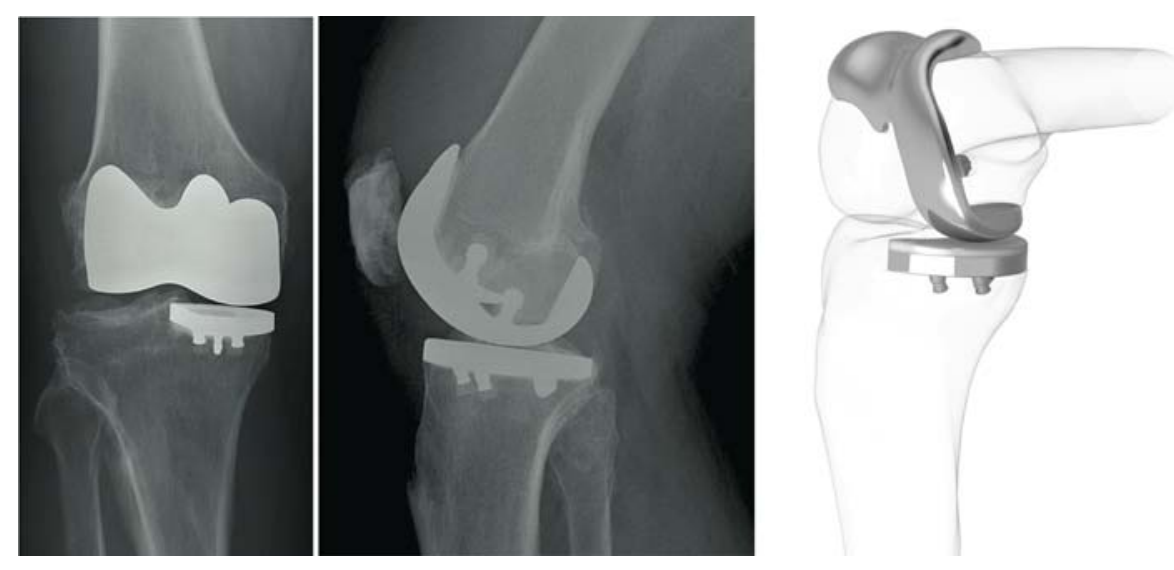

Figure 1: Post op and graphic images of the iDuo knee replacement

\section{Results}

Seven patients underwent bi-compartmental knee replacement using the iDuo system between 2013 and present time. The senior author performed all procedures. Average patient age was 60 (range 49-82) three of whom were female and four male. All but one patient have passed the one year follow up period, four have passed the two year stage and one has thus far been seen as an out-patient for over three years. Only 
Table 1a: Interval knee scores $(K S)$

\begin{tabular}{|l|l|l|l|l|l|l|l|l|l|l|l|}
\hline Pt. & Age & BMI & ASA & $\begin{array}{l}\text { Date of } \\
\text { surgery }\end{array}$ & $\begin{array}{l}\text { Pre-op } \\
\text { KS }\end{array}$ & $\begin{array}{l}\text { 6 week } \\
\text { KS }\end{array}$ & $\begin{array}{l}\text { 3 month } \\
\text { KS }\end{array}$ & $\begin{array}{l}\text { 6 month } \\
\text { KS }\end{array}$ & $\begin{array}{l}\text { 1year } \\
\text { KS }\end{array}$ & $\begin{array}{l}\text { 2 year } \\
\text { KS }\end{array}$ & $\begin{array}{l}\text { 3year } \\
\text { KS }\end{array}$ \\
\hline 1 & 58 & 28.2 & 2 & $12 / 02 / 14$ & 40 & 50 & 80 & 79 & 81 & 47 & \\
\hline 2 & 57 & 23.5 & 1 & $19 / 11 / 14$ & 48 & 48 & 90 & 90 & 97 & & \\
\hline 3 & 82 & 25.3 & 2 & $12 / 02 / 04$ & 65 & 85 & N/A & 87 & 86 & 92 & \\
\hline 4 & 57 & 29.3 & 2 & $03 / 12 / 14$ & 37 & 85 & 92 & 85 & 95 & & 95 \\
\hline 5 & 61 & 26.9 & 2 & $30 / 07 / 14$ & 74 & 80 & N/A & 87 & 98 & 95 & \\
\hline 6 & 55 & 23.9 & 1 & $26 / 08 / 15$ & 60 & N/A & 81 & 91 & & & \\
\hline 7 & 49 & 26.2 & 1 & $04 / 12 / 13$ & 75 & N/A & 94 & N/A & 97 & 100 & \\
\hline
\end{tabular}

Table 1b: Interval function scores (FS)

\begin{tabular}{|l|l|l|l|l|l|l|l|l|l|l|l|}
\hline Pt. & Age & BMI & ASA & $\begin{array}{l}\text { Date of } \\
\text { surgery }\end{array}$ & $\begin{array}{l}\text { Pre-op } \\
\text { FS }\end{array}$ & $\begin{array}{l}\text { 6 week } \\
\text { FS }\end{array}$ & $\begin{array}{l}\text { 3 month } \\
\text { FS }\end{array}$ & $\begin{array}{l}\text { 6 month } \\
\text { FS }\end{array}$ & $\begin{array}{l}\text { 1 year } \\
\text { FS }\end{array}$ & $\begin{array}{l}\text { 2 year } \\
\text { FS }\end{array}$ & $\begin{array}{l}\text { 3year } \\
\text { FS }\end{array}$ \\
\hline 1 & 58 & 28.2 & 2 & $12 / 02 / 14$ & 25 & 40 & 90 & 80 & 80 & 50 & \\
\hline 2 & 57 & 23.5 & 1 & $19 / 11 / 14$ & 50 & 70 & 90 & 95 & 100 & & \\
\hline 3 & 82 & 25.3 & 2 & $12 / 02 / 04$ & 40 & 50 & N/A & 70 & 100 & 70 & \\
\hline 4 & 57 & 29.3 & 2 & $03 / 12 / 14$ & 50 & 60 & 80 & 70 & 100 & & 100 \\
\hline 5 & 61 & 26.9 & 2 & $30 / 07 / 14$ & 60 & 60 & N/A & 100 & 100 & 100 & \\
\hline 6 & 55 & 23.9 & 1 & $26 / 08 / 15$ & 60 & N/A & 80 & 100 & & & \\
\hline 7 & 49 & 26.2 & 1 & $04 / 12 / 13$ & 70 & N/A & 80 & N/A & 100 & 100 & \\
\hline
\end{tabular}

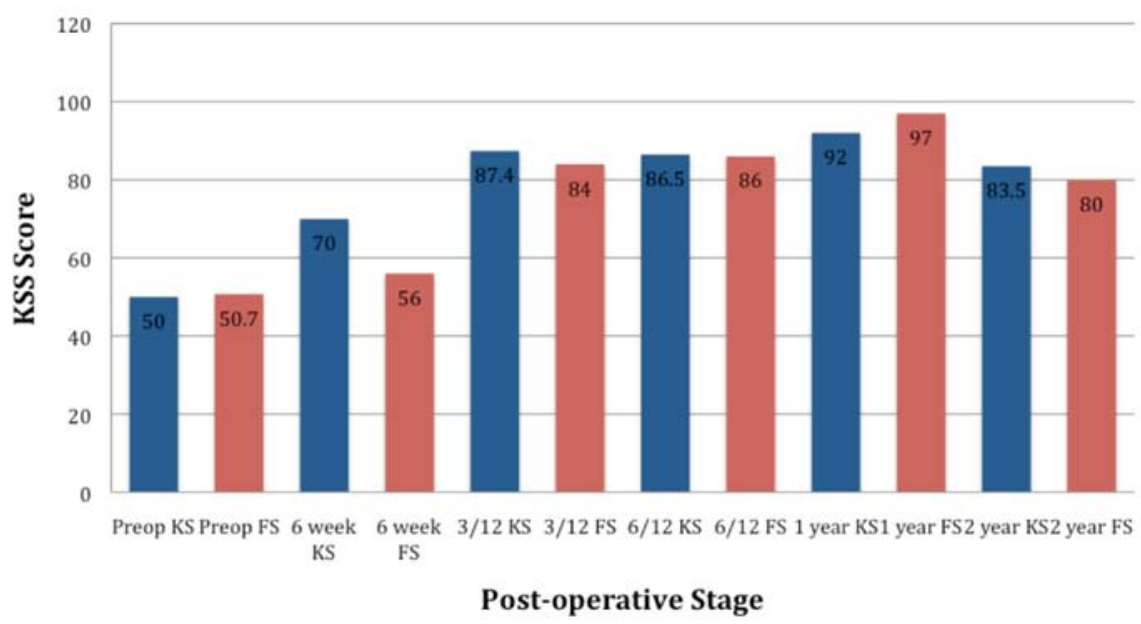

Figure 2: Average Knee \& Function Scores

one patient is retired. The remaining patients continue to work, three working in a clinical setting for the NHS. They all lead active lives, as reflected in part by body mass index (BMI) and American Society of Anesthesiologists scoring (ASA) shown in Table 1a\&b.

Scoring was not completed in two patients at 6 weeks, two patients at 3 months and in one patient at 6 months. Other than these omissions, the data set is complete (Table $1 \mathrm{a} \& \mathrm{~b}$ ). The average pre-operative KSS was 108 (knee score $57 /$ function score 51 ). These scores increased to reach excellent results above 80 in all cases (Table $1 \mathrm{a} / \mathrm{b} \&$ Figure 2). Where follow up was chronologically possible, we see that this outcome is maintained with the exception of one.

Patient 1 returned to theatre at 6 weeks for manipulation as her range of movement progression was deemed poor. She progressed well following this intervention and her scoring was excellent at 1 year. More recently she has developed increasing pain and stiffness as depicted in the Table 1 figures. Despite normal radiographs she is now under investigation for infection but as yet nothing abnormal has been found and she continues to work full time in the outpatients department. These particular scores have lead to the 2 year dip in Figure 2. Patient 3 has noticed a decline in function during the last year but attributes this to an arthritic contralateral knee. Other than patient 1 , all remaining patients would be willing to undergo the same operation again.

Interestingly, despite excellent outcomes even at 3 months, several patients subjectively felt that it took at least a year to start feeling satisfied with their recovery. Patient 4 previously underwent a unicondylar knee replacement in his contralateral knee. He feels that recovery with this particular knee was much quicker although long-term outcome has been subjectively similar to that of his iDuo knee. Morning stiffness/aching has been noted in most patients but only lasts a few steps. Knee scores have thus persistently failed to reach 100 .

\section{Discussion}

Amongst those undergoing TKR, it has been shown that $28 \%$ have a preserved lateral tibio-femoral joint [12]. The idea to replace only two compartments is attractive but by no means new $[\underline{13}, \underline{5}]$. Despite this, progression seems slow 
and evidence to support this concept is sparse. What is certain is that the arthroplasty cohort is becoming younger and has both higher functional demands and an increased chance of revision at a later date. Bone preservation and near normal joint kinematics should therefor be a priority.

The literature suggests relatively normal kinematics can be achieved by preserving both cruciates. PCL retention alone may lead to inconsistent femoral rollback [14] and paradoxical anterior translation in TKR during deep flexion [15]. In vivo bi-unicondylar knee studies have shown that their kinematics and stability are comparable with the native knee $[\underline{16}, \underline{17}]$. Wang et al [18] compared gait kinematics in BKA versus control limbs at 1 year. They showed little difference in walking performance, coronal plane mechanics and knee extensor moment. More recently this same group compared the iDuo knee with TKR patients and healthy controls. This study demonstrated that iDuo limbs possessed comparable strength, mechanics and speed to healthy controls during daily activities and that TKR limbs showed significant deficit to both healthy control subjects and iDuo limbs [19].

The Journey Deuce knee has been the only other monolithic design marketed but has now been withdrawn. Palumbo et al. converted $14 \%$ to total knee replacements at an average of 19 months for persistent pain. They found tibial base plate loosening as the cause. This could have been related to excess micro motion at the bone-cement interface [11]. Only $48 \%$ were found to have an excellent to good post - operative functional KSS. Morrison et al. revised 3 of 21 Journey-Deuce knees for persistent postoperative pain at one year and found an overall complication rate of $28.6 \%$ with BKA compared to $6.1 \%$ in their TKR cohort [20]. Tria et al. reported anterior knee pain in $26 \%$ of 40 knees and $12 \%$ needing revision after a mean follow up of 2 years [21] . All three papers reported tibial tray fractures.

Conversely, early data for the iDuo knee is more favorable. Bryant et al. reported that $91 \%$ of 34 knees at a mean follow up of 30 months had good to excellent results. Additionally, if given the choice to undergo the same operation again, 97\% replied yes. Our results seem to echo this sentiment but with the benefit of prospective knee outcome scoring. Thus far we have not recorded any of the complications associated with the Deuce knee and even if when including the data of patient 1 , all patients at 2 years have scores above 80 . The published results of modular BKA is a little more extensive. Both Paratte et al. []ㅡ and Heyse et al. [13] have reported long-term results with an average follow up of approximately 12 years. These papers suggest good to excellent functional and knee scores. Comparison with the iDuo knee is difficult as our cohort is small and follow up is short. This, we believe, reflects the age of the design and specific patient selection. There are currently no papers comparing modular and monolithic designs.

The question therefore, is why should the iDuo knee be any different? There are several design features, which may be key to its early success. The tibial tray is $2 \mathrm{~mm}$ thick and thus thicker than that of the Deuce knee leading to a lower risk of fracture. There is also an additional tibial component posterior keel for increased stability. The patient specific design may have several benefits when compared with "off the shelf" incremental size designs. Koeck et al. showed that tibial cortical fit/coverage is optimized and both component alignment and balance can be reliably achieved [22]. The Koeck paper was based on the ConforMIS iUni knee but the instrumentation, tibial tray features and design process for the implant is the same as that for the iDuo design. A patient specific femoral component not only preserves bone stock but also allows optimum geometry and alignment of both compartments. It is often difficult to gain this result with more standard designs, often achieving successful size and balance in one compartment but to the detriment of the other. Many times, we have to compromise in partial knee surgery, between fit of the implant and the alignment of the femoral and tibial interface. This can lead to edge loading on the tibial implant. The compromise is obviated with the iDuo system. By design, the fit will be specific for each patient's tibial and femoral condylar geometry and the contact area between the two will be maximized. These design features may render the iDuo knee at lower risk for loosening and pain in the future.

We mentioned earlier in this paper that it is a great shame to sacrifice healthy bone stock and the ACL when using TKR for bi-compartmental disease. Even though old age is not strict exclusion criteria for the iDuo, our patients are generally high functioning and below the age of 60 . It is therefore important to discuss outcomes in those who have undergone TKR at a younger age. After all, TKR would have been a perfectly acceptable option for our cohort.

Several recent papers cover TKR performed in younger patients. Meftah et al evaluated durability and functional outcome in patients who had undergone TKR and were 60 years and younger. At mean follow up of 12.3 years, overall survivorship was $98 \%$ and KSS was 80 and above in $95 \%$ of their fixed bearing knees [23]. Long et al have published results of 45 knees with an average of 25.1 years follow up and average age of 51. The average knee score was 87.4 and functional score. 62.1. In a similar but larger cohort (108 knees), survivorship without revision for any cause was $70.1 \%$ at 30 years [려]. Kim et al have showed that the KSS in TKR were excellent at 16.8 years in a co- 
hort with an average age of 45 years. The Kaplan-Meier survivorship for revision was 95\% [드] . These results would suggest that functional outcome and survivorship is good in younger patients who undergo TKR and function rivals that of our cohort. Survivorship cannot be compared. However, one advantage of the iDuo system compared with standard TKR may be ease of revision or better function thereafter as Bone stock preservation is a key feature of the iDuo knee.

There is no doubt that the limitation of this paper is that of patient number. However, the iDuo knee is relatively new to the market and may only be appropriate for a small population. Long-term data and comparison studies are required to further evaluate this knee prosthesis with particular attention to direct comparison with TKR.

\section{Conclusion}

Our early results suggest that the iDuo knee is a good option for those with isolated bi-compartmental disease and outcome scores are comparable with those reported for the BKA. This bicompartmental design may bridge the gap between the uni-compartmental and total knee replacement. The choice between monolithic or modular designs remains in debate. We will continue to use this prosthesis for a carefully selected group of patients.

\section{Disclosure}

The authors declare that there is no conflict of interest regarding the publication of this paper. For full disclosures refer to last page of this journal.

\section{References}

1. Hopper G, Leach W. (2008) Participation in sporting activities following knee replacement: total versus unicompartmental. Knee Surg Sports Traumatol Arthrosc 16:973.

2. Saccomanni B. (2010) Unicompartmental knee arthroplasty: a review of literature. Clin Rheumatol 29:339.

3. Temple MM, Bae WC, Chen MQ, et al. (2007) Age and site associated biomechanical weakening of human articular cartilage of the femoral condyle. Osteoarthritis Cartilage 15:1042.

4. Berger R, Meneghini R, Sheinkop M, et al. (2004) The progression of patellofemoral arthrosis after medial unicompartmental replacement: results at 11 to 15 years. Clin Orthop Relat Res;92
5. Parratte S, Pauly V, Aubaniac J- M, Argenson J- NA. (2010) Survival of bicompartmental knee arthroplasty at 5 to 23 years. Clin Orthop Relat Res 468:64-72.

6. Lonner JH. Modular bicompartmental knee arthroplasty with robotic arm assistance. (2009) Am J Orthop (Belle Mead NJ) 38 (suppl):28-31.

7. Parratte S, Ollivier M, Opsomer G, et al. (2015) Is knee function better with contemporary modular bicompartmental arthroplasty compared to total knee arthroplasty? Short-term outcomes of a prospective matched study including 68 cases. Orthop Traumatol Surg Res (Epub ahead of print) PMID: 26047754

8. Thienpont E, Price A. (2013) Bicompartmental knee arthroplasty of the patellofemoral and medial compartments. Knee Surg Sports Traumatol Arthrosc 21:2523-2531.

9. Yeo NE, Chen JY, Yew A, et al. (2015) Prospective randomised trial comparing unlinked, modular bicompartmental knee arthroplasty and total knee arthroplasty: A five years follow-up. Knee 22:321-327

10. Engh GA. (2007) A bi-compartmental solution: what the Deuce? Orthopedics 30:770- 771 .

11. Palumbo BT, Henderson ER, Edwards PK, et al. (2011) Initial experience of the Journey- Deuce bicompartmental knee prosthesis: a review of 36 cases. J Arthroplasty 26 (suppl):40-45.

12. Heekin RD, Fokin AA (2014) Incidence of bicompartmental osteoarthritis in patients undergoing total and unicompartmental knee arthroplasty: is the time ripe for a less radical treatment? J Knee Surg 27:77 82.

13. Heyse TJ, Khefacha A, Cartier P (2010) UKA in combination with PFR at average 12-year follow-up. Arch Orthop Trauma Surg 130:1227-30.

14. Dennis DA, Komistek RD, Colwell CE Jr, Ranawat CS, Scott RD, Thornhill TS, Lapp MA (1998) In vivo anteroposterior femorotibial translation of total knee arthroplasty: a multicenter analysis. Clin Orthop Relat Res 356:47-57

15. Stiehl JB, Komistek RD, Cloutier JM, Dennis DA (2000) The cruciate ligaments in total knee arthroplasty: a kinematic analysis of 2 total knee arthroplasties. J Arthroplast 15:545-550

16. Banks SA, Fregly BJ, Boniforti F, Reinschmidt C, Romagnoli S. (2005) Comparing in vivo kinematics of unicondylar and biunicondylar knee replacements. Knee Surg Sports Traumatol Arthrosc 13:551-556

17. Lo J, Müller O, Dilger T, Wülker N, Wünschel M (2011) Translational and rotational knee joint stability in anterior and posterior cruciate-retaining knee arthroplasty. Knee 18:491-495

18. Wang H, Dungan E, Frame J, Rolston L. (2009) Gait analysis after bicompartmental knee replacement. Clin Biomech 24:751-754

19. Wang H, Foster J, Francksen N, Estes J, Rolston L. (2015) Lunging after knee replacement surgeries. World Arthroplasty Congress (WAC)

20. Morrison TA, Nyce JD, Macaulay WB, Geller JA. (2011) Early adverse results with bicompartmental knee arthroplasty: a prospective cohort comparison to total knee arthroplasty. J Arthroplast 26:35-39

21. Tria A., et al. (2010) "Bicompartmental knee arthroplasty using a single piece femoral component";American Academy of Orthopaedic Surgeons Feb ; Abstract Podium 680

22. Koeck F et al. (2011) Evaluation of implant position and knee alignment after patient-specific unicompartmental knee arthroplasty . The Knee 18 ;294-299

23. Meftah M, White P, Ranawat A, Ranawat C (2016) Long-term results of total knee arthroplasty in young and active patients with posterior stabilized design. The Knee 23:318-321

24. Long et al. (2014) Total Knee Replacement in Young, Active Patients: Long-Term Follow-up and Functional Outcome. J Bone Joint Surg Am 96(18):e159(1-7)

25. Kim YH et al. (2012) Long-term comparison of fixed-bearing and mobile-bearing total knee replacements in patients younger than fifty-one years of age with osteoarthritis. J Bone joint Surg Am 94(10):866-73 